\title{
MAGNETIC SUSCEPTIBILITY OF MESOSCOPIC SYSTEMS
}

\author{
S. WALCERZ \\ Institute of Physics, Adam Mickiewicz University \\ J. Matejki 48/49, 60-769 Poznań, Poland
}

The Onsager description of de Haas-van Alphen oscillations and the magnetic translation group approach are combined in order to argue that the magnetic susceptibility of mesoscopic systems is assumed to reveal some features different than those of a bulk sample. Firstly, due to a discrete nature of magnetic field that has to be taken into account while dealing with the mesoscopic scale phenomena, the susceptibility should vary in a discrete way, too. Secondly, the susceptibility should depend on both the size and the shape of the system.

PACS numbers: 71.15.-m, 71.24.+q, 71.70.Di

\section{Origin of magnetic oscillations}

In the case of small systems the size effects and the finite Born-von Karman boundary conditions seem to play a role in the magnetic response of the system.

The explanation of the de Haas-van Alphen oscillations was done by Onsager [1]. Let $A\left(\epsilon_{\nu}\left(k_{z}\right), k_{z}\right)$ be the intersection of the Fermi surface with the plane perpendicular to the vector of the magnetic field $\boldsymbol{H}$. According to the Landau theory of free electron energy levels [2] the area $A$ is a function of $\nu$ - the number of the Landau level, and does not depend on the wave vector component $k_{z}$

$$
\begin{aligned}
& A\left(\epsilon_{\nu}\left(k_{z}\right), k_{z}\right)=A(\nu), \\
& A(\nu)=(\nu+\lambda) \Delta A
\end{aligned}
$$

for $\nu$ being large enough (of an order of the Avogadro number). In the case of free electrons $\lambda=1 / 2 . \Delta A$ describes the difference between the two areas: $A(\nu)$ and $A(\nu+1)$, corresponding to the two neighbouring levels $\nu$ and $\nu+1$, respectively. $\Delta A$ can be related to the value of the magnetic field parallel to the $z$-axis as:

$$
\Delta A=\frac{2 \pi e|\boldsymbol{H}|}{\hbar c}=\frac{2 \pi H}{\phi_{0}}
$$

where $\phi_{0}$ is the elementary flux of the magnetic field. It is essential that $A(\nu)$ depends linearly on $H$ via $\Delta A$

$$
A(\nu)=(\nu+\lambda) \frac{2 \pi H}{\phi_{0}} \text {. }
$$


The movement of an electron in a magnetic field results in phase effects [3]. The notion of the magnetic translation group (MTG), introduced by Zak [4] and Brown [5], describes the symmetry of the electron in a crystal lattice field and the homogeneous external magnetic field. The magnetic translation operators

$$
\tau_{x}=\exp \left\{\frac{\mathrm{i}}{h} a_{x}\left[p-\frac{e}{c} \boldsymbol{A}\right]_{x}\right\}, \quad \tau_{y}=\exp \left\{\frac{\mathrm{i}}{h} a_{y}\left[p-\frac{e}{c} A\right]_{y}\right\}
$$

act on the electron wave functions in a crystal lattice, for instance rectangular of constants $a_{x}, a_{y}$. The operators $\tau_{x}, \tau_{y}$ fulfill the relation

$$
\tau_{x} \tau_{y}=\tau_{y} \tau_{x} \epsilon
$$

where the phase factor $\epsilon$ is a complex number of the modulus equal unity. Because of the magnetic boundary conditions

$$
\epsilon^{N}=1
$$

the phase factor is of the form $\epsilon=\exp (2 \pi \mathrm{i} l / N)$, where $N$ is the Born-von Karman period, $l-$ an integer relatively prime with $N$. The requirement that the operators $\tau_{x}, \tau_{y}$ commute with the Hamiltonian

$$
\mathcal{H}=\frac{1}{2 m}\left[p+\frac{e}{c} \boldsymbol{A}\right]^{2}+V(\boldsymbol{R})
$$

(here $\boldsymbol{A}=\frac{1}{2}[\boldsymbol{H} \times \boldsymbol{r}]$ ) leads to the quantization condition (rationality condition)

$$
\frac{\boldsymbol{H} \cdot \boldsymbol{S}}{\phi_{0}}=\frac{l}{N} 2 \pi
$$

where $\boldsymbol{S}=\boldsymbol{a}_{x} \times a_{y}$. The physically valid representations of the magnetic translation group have to be found within the small magnetic Brillouin zone. The practical method of constructing and classifying all the physical representations of MTG has been worked out by Florek and Lulek [6].

The two presented approaches can be combined through the connection between $A-$ the area of a certain intersection of the Fermi surface, and $S$ - the area of the elementary crystal cell. The Onsager theory as well as the MTG formalism permit the derivation of an expression for the elementary flux $\phi_{0}$, although in different ways. In the Onsager theory

$$
\frac{H}{\phi_{0}}=\frac{1}{\nu+\lambda} \frac{A}{2 \pi}
$$

obtained using the approximation of the continuous Fermi surface $(N \rightarrow \infty)$. In the MTG formalism

$$
\frac{H}{\phi_{0}}=\frac{l}{N} \frac{2 \pi}{S}
$$

calculated assuming finite $N$.

Taking into account that $A(\nu)=$ const for a given $\nu$, one obtains

$$
A S=C, \quad C-\text { a numerical constant, }
$$

as $A$ is defined in a reciprocal lattice, while $S$ is in the simple one. Moreover, both models involve the universal quantity $\phi_{0}$, so one can compare (9) with (10) which yields

$$
\frac{1}{\nu+\lambda} C^{\prime}=\frac{l}{N}, \quad C^{\prime}=\frac{A S}{(2 \pi)^{2}} .
$$

Thus, the number $\nu$ of the Landau level has been related to a particular integer $l$. 


\section{Magnetic susceptibility}

Since electrons in metal particles constitute finite Fermi systems, e.g. the electrons exhibit saturation or constancy of particle density, the quantities characterizing the Fermi gas (like Fermi velocity) used to be employed for describing the phenomena occurring in small systems, too (see for example [7]). Therefore the formula (12) could be of some use while describing magnetic properties of a crystal, for example in determining the magnetic susceptibility

$$
\chi=\frac{1}{v B_{0}} \frac{e \hbar}{m c} \nu(\nu+1),
$$

where $B_{0}=2 \phi_{0} \pi n / L^{2}, v=V / n, V-$ volume of the crystal, $L-$ linear size of the crystal, $n-$ number of electrons. Thus, the susceptibility would be derived as $\chi=\left\{\frac{L^{2}}{V} \frac{1}{2 \pi \phi_{0}} \frac{e \hbar}{m c}\right\} \nu(\nu+1)=\{D\} \nu(\nu+1)=D\left(\frac{N}{l^{\prime}}-\frac{1}{2}\right)\left(\frac{N}{l^{\prime \prime}}+\frac{1}{2}\right)$,

where $l^{\prime}, l^{\prime \prime}$ - integers relatively prime with $N$.

The susceptibility oscillations could be then precisely determined using (14). In the case of the mesoscopic systems, for which the number $N$ is of an order of $10^{3}$, it is practically possible to take into account all the numbers $l^{\prime}, l^{\prime \prime}$ relatively prime with $N$. There could be also possible to verify experimentally the usefulness of the formula (14) with the help of the SQUID magnetometer, providing one had at one's disposal the isolated mesoscopic sample fulfilling the magnetic boundary conditions.

\section{References}

[1] L. Onsager, Philos. Magn. 43, 1006 (1952).

[2] L.D. Landau, Z. Phys. 64, 629 (1930).

[3] Y. Aharonov, D. Bohm, Phys. Rev. 115, 485 (1959).

[4] J. Zak, Phys. Rev. A 134, 1602, 1607 (1964); ibid. 136, 776 (1964).

[5] E. Brown, Phys. Rev. A 133, 1038 (1964).

[6] W. Florek, Rep. Math. Phys. 34, 81 (1994); T. Lulek, Rep. Math. Phys. 34, 71 (1994).

[7] R.S. Bhalerao, M. Barma, Pramana 40, 311 (1993). 